management for this group is coronary angiography within 24 hours. In our audit, $51 \%$ of patients reached angiography within 24 hours.

- Current use of multiple notes creates the potential for patient safety issues, integrating notes across disciplines would reduce this.

- Variation in discharge planning and advice, with delays to discharge to clarify outpatient follow up and discharge medications.

Interventions Immediate Intervention - educating the MDT regarding gold standard care, specifically screening for risk factors.

Short term intervention - introduction of a 'cardiac risk factor' screening sticker to improve risk factor identification over 1 month.

Long Term Intervention - NSTE-ACS multi-disciplinary pathway to include the medical notes, nursing notes and procedural notes if coronary angiography is undertaken. This will improve the efficiency of listing patients who meet the ESC criteria for 'high-risk NSTE-ACS' for urgent angiography.

By including a section on risk factor management, we hope to see a sustained improvement in screening for diabetes and dyslipidaemia.

Finally, it will include a summary of the patients' journey which can be translated accurately onto a discharge letter.

\section{IMPLEMENTING THE COVID-19 COMMUNICATIONS PATHWAY FOR JUNIOR DOCTORS AT BUCKINGHAMSHIRE HEALTHCARE NHS TRUST}

Hirushi Jayasekera, Ayah Babiker, Jennifer Abou Jawdeh. Buckinghamshire Healthcare NHS Trust, Oxford Deanery, UK

\subsection{6/leader-2020-FMLM.106}

In the midst of the pandemic, an issue was raised to the 'Junior Doctors' Forum' (JDF) that the high influx of emails consisting of vital information (pertaining to clinical practice etc.) was being missed due to a number of reasons. This led to uncertainty, confusion, and anxiety voiced by the Junior Doctor body. Our aim was to address the many queries of the doctors and streamline information in a structured and timesensitive manner. This project was discussed with the CEO, Medical Director, Director of Medical Education and Guardian of Safe working.

A questionnaire was sent out in early March to help identify the preferred mode of communication which was found to be though email. Intervention in the form of the 'Covid 19 Communication Pathway' was implemented Trust-wide. It links the senior management with front-line doctors directly paving the way for stronger working relationships and teamwork. Updated information was sent down the pathway and queries were simultaneously escalated up.

Post intervention, a second questionnaire was repeated in July 2020 seeking feedback regarding the effectiveness of the pathway. Positive feedback was also received by the CEO and the Medical Director in acknowledgement of the C19 Pathway being a formal mode of communication during the pandemic.
In terms of leadership, this project highlights the importance of liaising with multiple members of staff to ensure that everyone is working towards a common goal. It provides doctors with a reliable platform through which to escalate queries and concerns. It has helped allay a lot of staff anxiety stemming of uncertainties faced at the peak of the pandemic. Through providing our doctors with up-to-date platform of receiving the latest guidelines, management, protocols etc. we have enabled all our staff reliable access to information which will indirectly optimise the healthcare provided during this pandemic.

\section{Leading across systems and organisations}

\section{EXPLORING HOW RECEIVING A TAX BILL DUE TO THE ANNUAL ALLOWANCE TAPER OF THE NHS PENSION SCHEME HAS AFFECTED SURGEON'S PERCEPTIONS OF WORKING IN THE NHS; A QUALITATIVE STUDY}

Lydia Bancroft. University of Birmingham, Medical School, Edgbaston, Birmingham, B15 $2 \pi, U K$

\subsection{6/leader-2020-FMLM.107}

Background The Annual Allowance (AA) taper caused many doctors to pay to work though receiving tax bills on their pension.

Aim To explore how receiving a tax bill due to the AA taper of the NHS Pension Scheme affected surgeons' perceptions of working in the NHS.

Methods In 2020, 12 qualitative, semi-structured, one-to-one interviews were conducted with surgeons from six NHS trusts. Thematic analysis.

Findings Before a bill, most participants were content working in the NHS as they had financial equilibrium, job satisfaction, and promised pension remuneration. However, receiving a bill was unaffordable and stressful. As working risked triggering future bills, participants became risk averse to working in the NHS and so reduced their NHS work. Many felt guilty withholding patient care but several were frustrated with the government and felt that the government was taking advantage of doctors. All believed that it was the governments' responsibility to resolve problems caused by the taper in the NHS but many distrusted the government and felt voiceless. The NHS Pension was viewed as remuneration for high work done at low pay, and a reward for loyalty and commitment to the NHS. A pensions tax bill broke this expectation, leaving participants demoralised and with reduced incentive to keep contributing.

Conclusions Receiving a tax bill disrupted the financial equilibrium, job satisfaction, and pension remuneration that participants expected of working in the NHS. Some found it a final straw so were considering retiring early and reported reduced engagement. Others experienced less disruption but still felt undervalued, mistreated, and demoralised which contributed to increased frustrations with working in the NHS. Although the 2020 Budget gave a financial solution to the taper, it may not resolve perceptions that some doctors now have of working in the NHS. Future grievances may exacerbate these perceptions, causing a greater loss of workforce engagement. 